\title{
Rapid D-dimer testing as an adjunct to clinical findings in excluding pulmonary embolism
}

\author{
A F Lennox, A N Nicolaides \\ Irvine Laboratory for Cardiovascular Investigation and Research, Imperial College \\ School of Medicine at St Mary's Hospital, London, UK
}

Introductory article

\section{Usefulness of D-dimer, blood gas, and respiratory rate measurements for excluding pulmonary embolism}

\author{
P Egermayer, G I Town, J G Turner, D C Heaton, A L Mee, M E J Beard
}

Background. A study was undertaken to assess the usefulness of the SimpliRED D-dimer test, arterial oxygen tension, and respiratory rate measurement for excluding pulmonary embolism (PE) and venous thromboembolism (VTE). Methods. Lung scans were performed in 517 consecutive medical inpatients with suspected acute PE over a one year period. Predetermined end points for objectively diagnosed $P E$ in order of precedence were (1) a post mortem diagnosis, (2) a positive pulmonary angiogram, (3) a high probability ventilation perfusion lung scan when the pretest probability was also high, and (4) the unanimous opinion of an adjudication committee. Deep vein thrombosis (DVT) was diagnosed by standard ultrasound and venography. Results. A total of 40 cases of PE and 37 cases of DVT were $\vec{\circ}$ objectively diagnosed. The predictive value of a negative SimpliRED test for excluding objectively diagnosed PE was 0.99 (error rate 2/249), that of $\mathrm{PaO}_{2}$ of $\geqslant 80 \mathrm{~mm} \mathrm{Hg}(10.7 \mathrm{kPa}$ ) was 0.97 (error rate $5 / 160$ ), and that of a respiratory rate of $\leqslant 20 /$ min was 0.95 (error rate 14/308). The best combination $\leftrightarrows$ of findings for excluding $P E$ was a negative SimpliRED test and $\mathrm{PaO}_{2} \geqslant 80 \mathrm{~mm} \mathrm{Hg}$, which gave a predictive value of 1.0 (error rate 0/93). The predictive value of a negative SimpliRED test for excluding VTE was 0.98 (error rate 5/249). Conclusions. All three of these observations are helpful in excluding $P E$. When any two parameters were normal, PE was very unlikely. In patients with a negative SimpliRED test and $\mathrm{PaO}_{2} \geqslant 80 \mathrm{~mm} \mathrm{Hg}$ a lung scan is usually unnecessary. Applications of this approach for triage in the preliminary assessment of suspected PE could lead to a reduced rate of false positive diagnoses 9 and considerable resource savings. (Thorax 1998;53:830-4)

It is well established that clinical signs alone are unreliable in the diagnosis of both acute pulmonaryembolism (PE) and deep venous thrombosis (DVT) that the majority of patients who present with suspected venous thromboembolism (VTE) do not have the disease. In particular, symptoms and signs of PE such as respiratory rate and oxygen saturation may be indistinguishable from those of other cardiorespiratory disorders. In recent years, with the widespread availability of objective testing methods such as ventilation/ perfusion lung scanning, pulmonary angiography and spiral CT scanning, there are large numbers of patients undergoing negative complex investigations to exclude the presence of disease. Since the prevalence of PE ranges from less than 10\% (as in the study featured in the introductory articl of to $40 \%$ in patients where there is clinical suspicion, there have been many diagnostic strategies proposed to improve the sensitivity and speci- ficity of combinations of tests for patients requiring further investigation. These diagnostic algorithms usually involve one or more tests, with definitive treatment being started only when one of these is positive. @्? However, pulmonary angiography is invasive and the 0 other tests are both costly and time consuming. Thus, much interest has been shown in developing a simple test or combination of simple tests that could reliably $\stackrel{\mathbb{Q}}{\varrho}$ exclude PE and obviate the need for further assessment in the majority of patients. The authors of this article o have been able to show that a combination of normal respiratory rate and normal arterial blood gas oxygen tension can almost exclude the presence of PE. When combined with a simple, rapid whole blood D-dimer assay that can be performed and interpreted at the bedside, these measurements can have a 100\% negative predictive value - that is, the disease can be excluded in the presence of a negative test. This would appear 


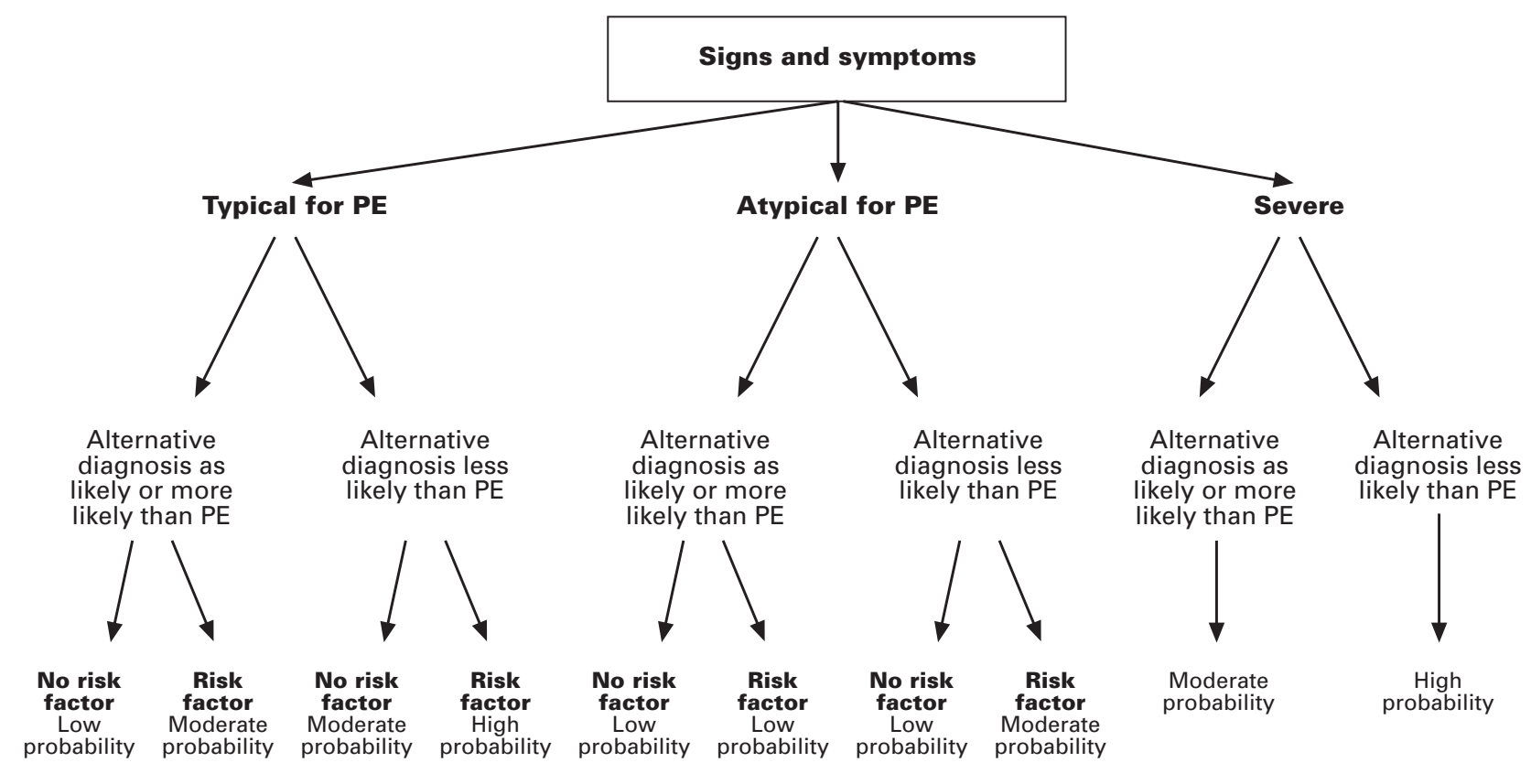

\begin{tabular}{|l|}
\hline Typical for PE \\
- Patient has $\geq 2$ respiratory points \\
and heart rate $>90 / \mathrm{min}$, leg \\
symptoms, low grade fever, \\
or chest radiograph compatible \\
with PE \\
Atypical for PE \\
- Patient has respiratory or cardiac \\
symptoms but does not meet \\
criteria for "typical" \\
Severe \\
- Patient meets "typical" definition \\
but also has \\
- Syncope, BP <90 mm Hg with heart \\
rate $>100 /$ min, receiving ventilation \\
or requires $>40 \%$ oxygen \\
supplementation, new onset \\
right heart failure (S1, Q3, T3 \\
or RBBB)
\end{tabular}

Respiratory points
- Dyspnoea or worsening of chronic
dyspnoea
- Pleuritic chest pain
- Arterial $\mathrm{O}_{2}<92 \mathrm{~mm} \mathrm{Hg}(12.3 \mathrm{kPa})$
on room air that corrects with
$<40 \%$ supplementation
- Haemoptysis
- Pleural rub

Respiratory points dyspnoea

- Pleuritic chest pain

on room air that corrects with

$<40 \%$ supplementation

- Pleural rub

Risk factors
- Surgery within 12 weeks
- Immobilisation for $>3$ days in
previous 4 weeks or paralysis
- Previous DVT or PE
- Lower limb fracture
- Strong family history of DVT or PE
- Malignancy within previous
6 months
- Postpartum

Risk factors

previous 4 weeks or paralysis

- Previous DVT or PE

- Malignancy within previous

- Postpartum

Figure 1 Algorithm for determining the probability of PE based on clinical assessment (modified from Wells et a

to be an attractive option in clinical practice with potential for both cost and time savings in terms of more complex investigations. However, the application of such an approach for widespread use in the hospital setting and its cost effectiveness is less well defined.

\section{Clinical assessment in pulmonary embolism}

Clinical signs are notoriously inaccurate in the diagnosis of lower limb DVT and PE as many other conditions can mimic thromboembolic disease. When taken in isolation, an increased respiratory rate is not a specific sign for PE, but its absence has been suggested as an effectivescreen for PE with a negative predictive value of $95 \%$ Of the 39 patients with objectively diagnosed PE, 14 (36\%) had a respiratory rate of less than 20/ min. This figure is supported by clinical data in the Prospective Investigation of Pulmonary Embolism Diagnosis (PIOPED) study where $30 \%$ of patients with confirmed PE had a respiratory rate of less than 20 breaths $/ \mathrm{min}$. By combining this figure with a normal arterial blood oxygen level they were able to increase the negative predictive value of a normal result to $98 \%$.

Wells et al have been able to stratify patients into differing 을. risk groups for PE based on a more extensive assessment $\mathrm{N}$ and combination of clinical symptoms and signs as or illustrated in fig 1. ${ }^{8}$ This group previously validated the $\stackrel{N}{\circ}$ utility of a pretest probability assessment in patients $\underset{\omega}{\mathbb{N}}$ with suspected DVT using a number of major and $\underset{2}{2}$ minor factors that were assigned different weightings of $\varphi$ clinical importanc $\Phi^{-11}$ and applied modifications of $\Phi$ these to the diagnosis of PE. By separating patients into differing risk groups prior to objective testing they were $\frac{0}{0}$ able to improve the diagnostic value of clinical assess- $\overrightarrow{\mathbb{D}}$ ment alone and identify those patients who had a low $\frac{\rho}{\mathbb{D}}$ risk for further thromboembolic complications over a $\Omega$ subsequent follow up period. It is these patients who could be included in diagnostic algorithms with $\mathrm{D}-$ o dimer testing to avoid diagnostic ventilation/perfusion scanning or invasive pulmonary angiography when scan- $\frac{\overline{0}}{7}$ ning is non-diagnostic. Whilst clinical assessment alone $\stackrel{\rightleftharpoons}{\rightleftharpoons}$ is not being proposed as the only method of diagnosis, by adopting a thorough risk stratification system based on the evidence in the literature, the clinician can more appropriately select those patients who require more invasive investigations to exclude PE. 


\section{D-dimer screening for exclusion of VTE}

D-dimer is a product of endogenous fibrinolysis and, although increased levels are found in acute DVT and $\mathrm{PE}$, they may also be raised in a number of other conditions such as infection, trauma, malignancy, and other inflammatory disorders. As a result the sensitivity and specificity of this assay is variable in different patient groups. Nevertheless, it has been shown in many studies that the presence of a normal D-dimer level can reliably exclude the presence of VTE with a predictive value greater than $95 \% 1^{21}$ Over the last decade the standard reference tests have been based on monoclonal antibody specific ELISA techniques with D-dimer levels greater than $500 \mathrm{ng} / \mathrm{ml}$ being considered abnormal. More recently there have been a number of latex and whole blood agglutination testing kits available. These have advantages over ELISA tests in that (1) they can be performed and interpreted more rapidly, (2) they do not require expensive laboratory equipment or technical expertise and do not need to be processed in batches to be cost effective, and (3) they are relatively inexpensive. These factors support the use of rapid testing kits as potential screening tools for the exclusion of both DVT and PE, particularly in the emergency setting, and the study by Egermayer et at 6 has demonstrated a negative predictive value of $99 \%$, at least comparable to most reported series.

The majority of studies have compared a single Ddimer testing method with a standard objective diagnostic test but some have compared several rapid latex and agglutination kits with traditional ELISA methods on the same patients. ${ }^{22}$ For example, the SimpliRED ${ }^{\text {TM }}$ whole blood agglutination test which has been available for several years and was used in the current study has been assessed widely with good sensitivity and negative predictive value for the exclusion of both DVT and PE. Although the result obtained is a subjective interpretation of the degree of agglutination in the drop of blood with the D-dimer antibody, the reported interobserver agreement, between-assay agreement, and reproducibility is greater than $95 \%{ }_{23}$ Despite these findings, Freyberger et $d l^{22}$ and Janssen et d $t^{21}$ were unable to demonstrate similar results when compared with the ELISA tests or other rapid latex tests. They found a sensitivity of $61-79 \%$, specificity of $67-90 \%$, and a negative predictive value of only $52-77 \%$, but it should be noted that the blood collection and storage techniques prior to analysis varied. These included the use of plasma samples that were later reconstituted with the addition of red cells which the authors acknowledge may have caused the reduced predictive values. The number of false positive $\mathrm{D}$-dimer results in the different patient groups (surgical vs medical, inpatients vs outpatients) will affect the specificity and positive predictive value of the test, but will reduce its clinical application to a much lesser degree providing the proportion of false negative tests remains low and the ability of the test to exclude VTE remains high. Those wishing to use the individual tests need to understand some of their limitations before using them in clinical practice. Some of the newer D-dimer testing kits may require extensive investigation before being adopted. Van Beek et a $f^{15}$ have shown that, for PE, acceptance of a test sensitivity of less than $100 \%$ may have serious consequences in that one per 1000 evaluated patients with clinically suspected PE would die for every $2 \%$ decrease in sensitivity if this was accepted

Brimble et af $\mathrm{F}^{4}$ and Reber et a ${ }^{25}$ have reported the importance of using a soluble fibrin assay in addition to the rapid D-dimer test to exclude VTE reliably. They

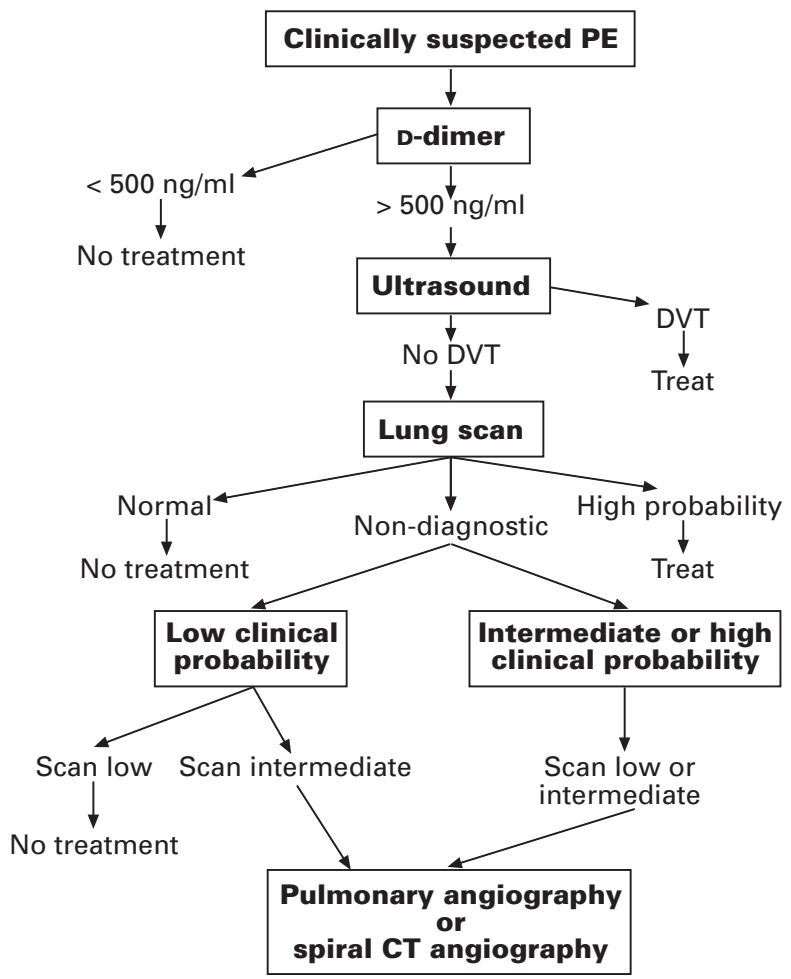

Figure 2 Proposed algorithm for the investigation of patients with clinically suspected PE based on cost effectiveness analysis (based on Perrier et a $\left.\right|^{2}$ ).

have proposed this as needing further evaluation because up to $10 \%$ of their patients with acute DVT or PE have had normal SimpliRED D-dimer results, impaired fibrinolysis possibly accounting for the negative result. Soluble fibrin assays are not available for rapid bedside investigation at present. However, if developed, they may complement D-dimer tests as useful screening tools.

\section{Cost effectiveness of rapid D-dimer testing}

Several diagnostic algorithms have been proposed for $\frac{0}{3}$ the investigation of patients with suspected VTE and $\dot{8}$ some of these have been validated in large studies assessing safety, reliability, and recurrence rates. Most 을 of these require tests that are not always available at all times or in all centres, particularly late at night or at weekends, and patients are often started on anti- $\bar{N}$ coagulation before a definitive diagnosis is made to $\mathrm{N}$ prevent early complications and progression of VTE. $\stackrel{\sim}{\circ}$ This exposes large numbers of patients to the risks of $\mathrm{N}$ anticoagulation and is wasteful of hospital resources. Although the current studf does not address cost $\bullet$ effectiveness, it has been shown by Perrier et d $l^{2}$ that $\mathbb{\Phi}$ the use of non-invasive diagnostic techniques can save $\stackrel{+}{+}$ time and money. By using D-dimer and lower limb 꿍 duplex ultrasonography in combination with ventilation/ perfusion lung scanning, a $9 \%$ incremental cost saving and a $47 \%$ decrease in pulmonary angiograms was achieved when angiography was only performed in those with an inconclusive non-invasive diagnostic work-up ${ }^{26}$

\section{Clinical applicability}

With negative predictive values of nearly $100 \%$, D-dimer measurement alone could be considered as the only screening test necessary. However, most authors have been reluctant to propose such a diagnostic strategy. Despite numerous reports that have prospectively as- 


\section{LEARNING POINTS}

* For patients with suspected pulmonary embolism clinical signs alone are unreliable in making the diagnosis.

* In the presence of low levels of D-dimer the diagnosis of pulmonary embolism is highly unlikely.

* Not all D-dimer testing methods have equal sensitivity or specificity, however the negative predictive value of the rapid bedside kits which approach $100 \%$ are comparable to the ELISA tests.

* By combining clinical parameters and D-dimer test results a safe and reliable investigation algorithm can be applied, resulting in a large proportion of ventilation/perfusion scans $\vec{\circ}$ being avoided.

* The use of D-dimer testing is time and cost effective.

sessed the reliability and accuracy of all forms of Ddimer assay, there is little evidence that clinical practice has altered significantly. Large cohorts of patients with clinically suspected VTE have been compared using reference investigations (lower limb venography and either pulmonary angiography or ventilation/perfusion scanning) and the use of D-dimer measurement alone or in combination with the clinical pretest probability. Both have proved reliable and safe. Various algorithms for investigating patients with suspected VTE have been proposed (fig 2). Although applicable in the majority of patients resulting in significant cost savings due to reduction in the number of unnecessary angiograms, lower limb duplex scans, ventilation/perfusion scans or spiral CT scans being performed, there remains a reluctance to follow this type of approach. This may be due to the concern physicians have regarding the absolute certainty of a negative D-dimer test on further management and outcome. There are both ethical and medicolegal issues involved in misdiagnosis of VTE which are at the forefront of the physician's mind.

We believe that $\mathrm{D}$-dimer testing will have a key role in future clinical practice, particularly in the emergency setting, allowing patients who are at low clinical risk of VTE with a negative D-dimer assay to be spared immediate hospital admission, anticoagulation, and investigation. Also, in situations when the patient presents after working hours or at weekends when the full range of diagnostic tests may not be available, a negative Ddimer test would allow the patient to be discharged until further non-invasive tests could be performed the following day, thereby avoiding hospital admission and anticoagulation.

1 Criado $\mathrm{E}$, Burnham CB. Predictive value of clinical criteria for the diagnosis of deep vein thrombosis. Surgery 1997;122:578-83.

2 Nypaver TJ, Shepard AD, Kiell CS, et al. Outpatient duplex scanning for deep vein thrombosis: parameters predictive of a negative study result. F Vasc Surg 1993;18:821-6.

3 McLachlin J, Richards T, Paterson JC. An evaluation of clinical signs in the diagnosis of deep vein thrombosis. Arch Surg 1962;85:738-44

4 Cranley JJ, Canos AJ, Sull WJ. The diagnosis of deep venous thrombosis: fallibility of clinical symptoms and signs. Arch Surg 1976;111:34-6.

5 Sandler DA, Duncan JS, Ward P, et al. Diagnosis of deep vein thrombosis: comparison of clinical evaluation, ultrasound plethysmography and venoscan with $\mathrm{x}$-ray venogram. Lancet 1984;ii:716-9.
6 Egermayer P, Town G, Turner J, et al. Usefulness of D-dimer, blood gas, and respiratory rate measurements for excluding pulmonary gas, and respiratory rate measurem

7 Worsley D, Alavi A. Comprehensive analysis of the results of the PIOPED study. F Nucl Med 1995;36:2380-7.

8 Wells P, Ginsberg J, Anderson D, et al. Use of a clinical model for safe 윽 management of patients with suspected pulmonary embolism. Ann Intern Med 1998;129:997-1005.

9 Wells PS, Hirsh J, Anderson DR, et al. Accuracy of clinical assessment of deep vein thrombosis. Lancet 1995;345:1326-30.

10 Wells PS, Hirsh J, Anderson DR, et al. A simple clinical model for the diagnosis of deep vein thrombosis combined with impedance $\mathscr{S}$ plethysmography: potential for an improvement in the diagnostic process. F Intern Med 1998;243:15-23.

11 Wells PS, Anderson DR, Bormanis J, et al. Value of assessment of pretest probability of deep vein thrombosis in clinical management. Lancet 1997;350:1795-8.

12 Duet M, Benelhadj S, Kedra W, et al. A new quantitative D-dimer assay appropriate in emergency: reliability of the assay for pulmonary embolism exclusion diagnosis. Thromb Res 1998;91:1-5.

13 Ginsberg J, Wells P, Kearon C, et al. Sensitivity and specificity of a rapid whole-blood assay for D-dimer in the diagnosis of pulmonary embolism. Ann Intern Med 1998;129:1006-11.

14 Tardy B, Tardy-Poncet B, Viallon A, et al. Evaluation of D-dimer ELISA test in elderly patients with suspected pulmonary embolism. Thromb Haemost 1998;79:38-41.

15 Van Beek E, Schenk B, Michel B, et al. The role of plasma D-dimer concentration in the exclusion of pulmonary embolism. Brf Haematol $\frac{\vec{F}}{0}$ 1996;92:725-32.

16 Wells PS, Brill EP, Stevens P, et al. A novel and rapid whole-blood assay for D-dimer in patients with clinically suspected deep vein thrombosis. Circulation 1995;91:2184-7.

17 Scarano L, Bernardi E, Prandoni P, et al. Accuracy of two newly ্ֻ㐅 described D-dimer tests in patients with suspected deep venous described D-dimer tests in patients

18 Bounameaux $\mathrm{H}$, deMoerloose P, Perrier A, et al. Plasma measurement of D-dimer as diagnostic aid in suspected venous thromboembolism: an overview. Thromb Haemost 1994;71:1-6.

19 Turkstra F, Van-Beek EJ, ten-Cate JW, et al. Reliable rapid blood test for the exclusion of venous thromboembolism in symptomatic 윽 outpatients. Thromb Haemost 1996;76:9-11.

20 Reber G, Coquoz C, de Moerloose P, et al. Comparison of two rapid D-dimer tests (Simplired and Vidas-DD) to rule out venous thromboembolism (abstract). Thromb Haemost 1997; June Suppl:158.

21 Janssen M, Heebels A, deMetz M, et al. Reliability of five rapid D- N dimer assays compared to ELISA in the exclusion of deep venous thrombosis. Thromb Haemost 1997;77:262-6.

22 Freyberger G, Trillaud H, Labrouche S, et al. D-dimer strategy in thrombosis exclusion. A gold standard study in 100 patients suspected $\boldsymbol{\omega}$ of deep venous thrombosis or pulmonary embolism; 8 D-dimer

23 Turkstra F, Van-Beek EJ, Büller HJ. Observer and biological variation of a rapid whole blood D-dimer test. Thromb Haemost 1998;79:91-3.

24 Brimble K, Ginsberg J. Evaluation of the combination of a bedside Ddimer assay and enzyme-linked immunosorbent soluble fibrin assay in patients with suspected venous thromboembolism. Thromb Res $1997 ; 88 \cdot 291-7$.

25 Reber G, Bounameaux H, Perrier A, et al. Performance of the fibrin monomer test for the exclusion of pulmonary embolism in symptomatic outpatients. Thromb Haemost 1999;81:221-3.

26 Perrier A, Buswell L, Bounameaux H, et al. Cost-effectiveness of noninvasive diagnostic aids in suspected pulmonary embolism. Arch Intern Med 1997;157:2309-16. 